\title{
Redes de colaboración entre dramaturgos en el teatro español del Siglo de Oro: nuevas perspectivas digitales
}

\section{Collaborative Networks Between Playwrights in Golden Age Spanish Theatre: New Digital Perspectives}

\section{Elena MARTÍNEZ CARro}

Universidad Internacional de La Rioja Avenida de La Paz, 137. Logroño, 26006 elena.martinez@unir.net

Orcid ID 0000-0001-6414-1724

\section{Alejandra Ulla LORENZO \\ Universidad Internacional de La Rioja Avenida de La Paz, 137. Logroño, 26006 alejandra.ulla@unir.net \\ Orcid ID 0000-0002-8137-9969}

Resumen: El objetivo del presente artículo consiste en proponer una metodología a través de la cual podamos analizar, haciendo uso de la teoría de redes o grafos, las relaciones sociales que se establecen entre los dramaturgos áureos que participan en la composición de comedias en colaboración. Se pretende, así, alcanzar conclusiones generales sobre el funcionamiento de la red de autores, la posición e importancia de estos en el contexto del grupo, las relaciones que se establecen entre ellos, así como las diferentes comunidades que se crean y su funcionamiento. Los resultados de dicho análisis ofrecerán una representación visual de los datos sobre la red recopilados en forma de grafo. La visualización de redes nos ayuda a mostrar patrones de relación o comunidades que no es posible determinar si estudiamos los textos de forma individual y, por lo tanto, a proponer conclusiones a partir de la información extraída de grandes grupos de textos o documentación histórica.

Palabras clave: Teatro del Siglo de Oro. Comedia en colaboración. Dramaturgo. Red. Grafo.
Abstract: This article proposes a method, based on the theory of nets or graphs, for analysing the social relations established between the Golden Age playwrights who take part in the composition of plays in collaboration. We pretend to achieve general conclusions on the operation of the net of authors, the position and importance of these in the context of the group, the relations established between them, the different communities that emerge and their operation. The results of this analysis will offer a visual representation of the data on the net in form of a graph. The visualization of nets helps us to show patterns of relation or communities that is not possible to determine if we studied the texts of individual form and, therefore, to propose conclusions from the information extracted of big groups of texts or historical documentation.

Keywords: Golden Age Theatre. Collaboration Play. Playwright. Network. Graph. 


\section{LAS COMEDIAS ESCRITAS EN COLABORACIÓN EN EL CONTEXTO CULTURAL DEL SigLO DE ORO}

L as novedades literarias del Siglo de Oro español fueron aparejadas a la calidad que encumbró a muchos de nuestros literatos y dramaturgos. La revolución teatral que Lope introdujo con el Arte Nuevo de hacer comedias encontró un ambiente propicio para la creación de un teatro genuino y propio que ha perdurado hasta nuestros días, no solo como reliquia de un pasado, sino como fuente de inspiración, renovación e investigación.

La corte de Felipe IV se constituyó en un centro de atracción de poetas y cómicos, al mismo tiempo que, en los ambientes populares, la afición al teatro crecía como principal distracción y fuente crematística. Dentro de este complejo panorama del teatro áureo surgieron -alrededor de 1630- las comedias colaboradas, compuestas entre varios poetas dramaturgos. A pesar del éxito que estas comedias tuvieron desde mediados del siglo XVII la crítica dieciochesca y la decimonónica las sepultó en el olvido (Mackenzie 37).

El modelo ha sido estudiado a lo largo del siglo XX desde distintas perspectivas, aunque quedan todavía algunos aspectos indefinidos y controvertidos. En las últimas décadas la academia ha intentado estudiar cuáles fueron las causas que originaron este tipo de colaboraciones para delimitar las intencionalidades artísticas y literarias de las puramente comerciales o circunstanciales. En este sentido es conocida la anécdota que publicó Pérez de Montalbán en 1636 en la Fama póstuma a la vida y muerte del doctor fray Lope Félix de Vega Carpio que -para muchos de los investigadores- justificaría el origen de las obras escritas en colaboración (33). Según esta anécdota las comedias mancomunadas nacieron para atender las urgencias teatrales. La composición entre varios ingenios permitía satisfacer de manera más rápida la fuerte demanda. Sin embargo, fue Mackenzie quien primero puso en duda esta hipótesis sobre la génesis de las comedias colaboradas al detectar que sus temáticas se basaban -mayoritariamente- en refundiciones de obras antiguas con argumentos conocidos por los dramaturgos y el público contemporáneo. El conocimiento de la trama por el grupo de poetas permitía una reelaboración singular sobre el tema y un reparto de la comedia equitativo (Mackenzie 35). Desde esta perspectiva puede interpretarse que el origen de las comedias colaboradas fuera más un juego que una necesidad. No cabe duda, en todo caso, de que estas obras nacieron alrededor de un grupo de poetas relacionados entre sí y con vínculos de amistad; esto es, aquellos autores áureos que participaron en aca- 
demias, justas y vejámenes literarios en los que colaboraban de manera conjunta y que favorecían las relaciones de manera lúdica.

Así pues, es un hecho la participación de todos los dramaturgos -a partir de 1630- en este usus scribendi y, en efecto, puede decirse que no hay escritor teatral que no componga comedias junto a otros, hasta el punto de poder agruparlos por sus numerosas colaboraciones. Tradicionalmente, la relación de autores que colaboraron en la creación de comedias hasta finales del siglo XVII se ha considerado paralela a la llamada "escuela de Calderón", autores que de una u otra forma convivieron con él y compartieron su forma de hacer teatro. El declinar de la escuela lopesca dejó paso a un joven Calderón que gozaba del éxito y prestigio entre la corte y el público del momento, y que también supo tener sus seguidores entre los que se encuentran la mayoría de los dramaturgos colaboradores. Si bien es cierto que Calderón fue un posible punto de unión entre muchos de los dramaturgos de la segunda mitad del XVII, hay que señalar que algunos de ellos no intervinieron nunca con el ingenio, aunque tuvieran amistad, y sí lo hicieron con Moreto, Matos Fragoso, Cáncer o Belmonte, principales autores colaboradores del momento. A pesar de que sus comedias en colaboración no siempre se han considerado de gran calidad, sus obras expresan y sintetizan los modelos imperantes de una época, y reflejan la red social en la que se desenvolvieron y convivieron.

Las combinaciones amistosas en la producción de estas comedias son múltiples, hasta un total de ochenta y tres, incluyendo aquellas comedias donde colaboró un dramaturgo de fama con un ingenio de la corte desconocido. Algunas colaboraciones reflejan los grupos de colaboraciones más cercanas como las de Moreto, Matos Fragoso y Cáncer, que a su vez colaboraron con Martínez de Meneses, Rosete, Villaviciosa y Juan Bautista Diamante, o las generadas en torno a Rojas Zorrilla con Calderón, Coello y Vélez de Guevara.

\section{LA CRÍTICA ANTE LAS COMEDIAS COLABORADAS: LOGROS Y RETOS}

Aunque quizás las comedias colaboradas no fueron de gran calidad, la genialidad para dar forma y unidad a una obra escrita entre varios, y tener éxito en las tablas y en la imprenta, ha supuesto un aliciente constante para los investigadores áureos. En los últimos años se han estudiado minuciosamente las temáticas teatrales. Asimismo, resultan abundantes los trabajos sobre comedias colaboradas que han pasado a analizarse de manera independiente con el fin de comprender la 'oficina poética' en su posible génesis. De hecho, son estas investigaciones las que dejan patentes los problemas de autoría de estas comedias 
(Rodríguez-Gallego), pero también las relaciones y agrupaciones más reveladoras entre los poetas dramáticos del momento. ${ }^{1}$ A pesar de los importantes avances en la investigación, el fenómeno plantea todavía numerosos interrogantes que comprenden las dudas sobre su catalogación, formas de escritura y composición, intencionalidad y finalidad, principales dramaturgos que colaboraron entre sí, así como las redes sociales que existían entre ellos. Los estudios tradicionales sobre las comedias escritas en colaboración se han inclinado por atender a problemas particulares que surgen en este contexto de acuerdo con lo mencionado. Sin embargo, estas perspectivas aisladas en torno a autores y comedias han impedido ver y estudiar en su justa medida a los verdaderos protagonistas de las comedias en colaboración, por lo que resultaba necesario ofrecer un acercamiento global a la red social que integraban los dramaturgos que participaban en este fenómeno creativo. Los avances en los estudios de redes sociales, la progresiva, aunque no definitiva, fijación de un corpus de comedias en colaboración, gracias al trabajo de la crítica, y la ayuda de las tecnologías nos permiten estudiar de manera conjunta las relaciones que existieron entre estos poetas áureos así como su protagonismo en la segunda mitad del siglo XVII. Este tipo de análisis complementa y cuestiona los numerosos estudios llevados a cabo desde la perspectiva filológica y hermenéutica.

Dada esta circunstancia, el objetivo del presente artículo consiste en proponer una metodología para el análisis de las relaciones sociales que se establecen entre los dramaturgos áureos que participan en la composición de comedias en colaboración. Para alcanzar este propósito partiremos de la teoría de redes, cuyos fundamentos se explican más adelante. Se pretende así alcanzar conclusiones generales sobre el funcionamiento de la red de autores, la posición e importancia de estos en el contexto del grupo, las relaciones que se establecen entre ellos, así como las diferentes comunidades que se crean y su funcionamiento.

\section{TEORÍA Y ANÁLISIS DE REDES SOCIALES EN EL CAMPO DE LAS HUMANIDADES}

La creación de redes sociales, en tanto que estructuras de vínculos, supone un fenómeno que ha existido desde el nacimiento de conjuntos de personas que conviven bajo una serie de normas comunes. Con la finalidad de poder comprender las relaciones que se establecían entre los miembros de una red social se creó el

1 Recuérdense, entre otros, varios de los trabajos publicados en el volumen La comedia escrita en colaboración en el teatro del Siglo de Oro editado por Matas Caballero. 
análisis de redes mediante la teoría de grafos. Su objetivo fundamental consiste en averiguar la estructura de una red concreta así como la centralidad y conectividad de cada uno de sus miembros en función de su posición (Rodríguez Treviño 104). El resultado obtenido se ofrece en forma de visualización, esto es, a través de una representación visual de los datos en forma de grafo. Este consiste en un grupo de elementos denominados nodos que están relacionados a través de una serie de enlaces a los que llamamos aristas; así pues, esta representación nos permite obtener un panorama global de la red que estemos estudiando.

La visualización de redes nos ayuda a mostrar patrones de relación o comunidades que no es posible determinar si estudiamos los textos de forma individual. Por lo tanto, nos permite proponer conclusiones a partir de la información extraída de grandes grupos de textos o documentación histórica a los que no sería posible llegar mediante la lectura individual de dichas fuentes. Este tipo de aproximación a los datos responde al concepto de lo que Moretti (11-12) ha denominado distant reading o macroanálisis, lo que supone que se preste atención a los patrones o características generales más que a los detalles de un conjunto amplio de datos. La recopilación de información a gran escala en el campo de las humanidades y, concretamente, su extracción, evaluación, descripción, tratamiento y su visualización para poder observar patrones y dinámicas de creación, funcionamiento y disolución en las distintas comunidades son algunas de las preguntas que Suárez plantea en tanto que objetivos de la agenda de investigación de las llamadas humanidades digitales.

La reflexión teórica sobre las redes sociales proviene del siglo Xx y ha llegado hasta nosotros de la mano de autores de disciplinas como la psicología social o las matemáticas; aunque los primeros análisis informatizados no se llevaron a cabo hasta principios del siglo XXI (Azam y Federico 3). Salvo en lo que concierne al campo de la antropología, esta teoría apenas se ha aplicado al estudio de las humanidades. A este respecto puede recordarse el análisis de redes sociales aplicadas al contexto de la historia del libro y, concretamente, al mercado editorial y las diferentes figuras que forman parte de este. En este sentido debe tenerse en cuenta la reciente publicación internacional bajo la edición de Hinks y Feely que lleva por título Historical Networks in the Book Trade. A su lado, pueden considerarse los trabajos que Juan Luis Suárez ha impulsado desde el Seminario Cultureplex ${ }^{2}$ que dirige en la universidad de Wes-

2 Seminario Culturplex: http://www.cultureplex.ca/ (Fecha de la consulta: 15/01/2019). 
tern Ontario, centro desde el cual se ha estudiado el Barroco hispánico en tanto que formación cultural y humana complicada caracterizada por un conjunto de relaciones igualmente complejas, cuyo estudio se propone desde la acumulación de una gran cantidad de datos en lugar de hacerlo a partir del dato particular (Becerra Mayorga y Camacho Machado 115). Entre los distintos proyectos que han llevado a cabo resulta particularmente significativo aquel que tiene como objetivo el estudio de las relaciones que se establecen entre los agentes del mercado editorial ibérico del Siglo de Oro a través de la información que ofrecen los textos preliminares de los libros antiguos y, particularmente, las censuras y dedicatorias (Becerra Mayorga y Camacho Machado) así como la contenida en las portadas de dichos impresos (Brown, Soto-Corominas y Suárez). También debe mencionarse la labor inaugurada desde el proyecto BIESES que, recientemente, se ha centrado en el estudio de las autoras españolas anteriores al siglo XIX y sus redes de sociabilidad. ${ }^{3}$ Finalmente, pueden recordarse los trabajos que, en esta misma línea, se han publicado en el campo de la literatura y, concretamente, mediante la exposición visual de las relaciones que se establecen bien entre los miembros de una comunidad literaria (Van Vugt), bien entre los personajes que protagonizan una obra literaria (Isasi). Nuestro trabajo pretende, pues, inscribirse en esta reciente línea de trabajo en la que se hace uso del análisis de redes sociales para la investigación en el campo de las humanidades y, concretamente, del teatro áureo. En este campo trazaremos la red de colaboraciones entre aquellos dramaturgos que participaron en el fenómeno de la composición de comedias de consuno.

\section{Propuesta de CORpus de las COMEdias EN COLABORACión}

De manera previa a la recreación y análisis de la red es imprescindible recoger "datos sobre todos los actores que integran la red y sobre todas las posibles relaciones entre estos" (Rodríguez Treviño 119). A este respecto, en nuestro caso debe considerarse que el primer paso consistía en el trazado del corpus de las comedias escritas en colaboración, lo que entraña varias dificultades, particularmente en aquellos casos en los que no se ha conservado manuscrito autógrafo. En este sentido pueden recordarse las peculiaridades en el modo de transmisión de estas piezas que, a menudo, fueron excluidas de las partes de comedias indi-

3 Bieses: http://www.bieses.net/las-autoras-y-sus-redes-de-sociabilidad/ (Fecha de la consulta: $15 / 01 / 2019)$. 
viduales y publicadas en partes de varios autores o en sueltas (Ulla Lorenzo 7998); por lo que en muchas ocasiones sufrieron, si cabe, más que las comedias de un solo autor, problemas editoriales de todo tipo, entre los que se encuentran, como anteriormente hemos señalado, los vinculados con las falsas autorías.

Más allá de esta problemática debe considerarse cómo, en los últimos años, los avances en el campo de la edición crítica, que han favorecido el estudio de los textos con mayor profundidad, así como la aplicación de la estilometría al estudio de los textos del Siglo de Oro, han permitido poner en tela de juicio la atribución autorial tradicional algunos textos.

Dadas estas circunstancias hemos configurado el corpus a partir del elenco que ofrece la monografía en torno a los manuscritos autógrafos de las comedias en colaboración de Alviti, que hemos complementado y modificado a partir de algunas publicaciones posteriores. Alviti recoge un total de 145 comedias en colaboración (179-94). A este número total hemos añadido cinco comedias: La fingida Arcadia compuesta probablemente por Calderón de la Barca, Agustín Moreto y un tercer autor desconocido (Trambaioli); El privilegio de las mujeres a cargo de Rojas, Calderón y Antonio Coello (Vega GarcíaLuengos 187-90); La muerte de Valdovinos compuesta por Cáncer, Rojas y Vélez (Madroñal 55) y El prodigio de Alemania probablemente escrita entre Calderón y Antonio Coello (Vega García-Luengos 191-98). Del cómputo total hemos eliminado, sin embargo, El más impropio verdugo pues, de acuerdo con su editora (Álvarez Brito), la comedia es solo de Rojas Zorrilla.

En este estudio hemos modificado la autoría de algunas piezas con respecto a lo establecido por Alviti. Así, El hijo pródigo, atribuida inicialmente a tres ingenios, se adjudica ahora a Cáncer, Moreto y Matos Fragoso (Gavela); finalmente se añade Matos Fragoso a la composición de La fuerza del natural junto con Cáncer y Moreto (García Reidy 467-69) y se elimina la participación de Juan Coello en El robo de las sabinas, compuesta por Rojas Zorrilla y Antonio Coello (González Cañal 113).

\section{RECREACIÓN DE LA RED SOCIAL DE DRAMATURGOS PARTÍCIPES EN EL FENÓMENO COLABORATIVO}

Desde ese punto de partida hemos podido crear, en primer lugar, una tabla en la que se recogen los 76 nodos integrados por los dramaturgos que participaron en la composición de 149 comedias en colaboración; y, en segundo, una de aristas. En ella se incluyen las relaciones que se establecen entre cada nodo a partir de sus interacciones indirectas en la creación de las piezas teatrales, 
que asciende a 193, y el número de colaboraciones total que se establece entre cada uno de ellos.

En la actualidad son muchas las herramientas de visualización que podemos emplear con el objetivo de mostrar gráficamente los resultados de nuestro estudio y que permiten trabajar con un volumen amplio de datos. En nuestro caso hemos manejado Gephi, en su versión 0.9.2, para el procesamiento de los datos, un software de acceso abierto que permite el análisis y la visualización de redes sociales. Con ayuda del programa es posible llevar a cabo de forma automatizada una serie de cálculos matemáticos de acuerdo con varios conceptos que nos permiten estudiar la posición de los nodos en la red y las propiedades de la red completa tal y como se observa en la siguiente tabla:

\begin{tabular}{|c|c|c|c|}
\hline LABEL & Degree Centrality & Betweenness Centrality & EIGENVECTOR CENTRALITY \\
\hline 1 Juan de Matos Fragoso & 50 & 438702 & 1 \\
\hline 2 Jerónimo de Cáncer & 33 & 195167 & 0,606565 \\
\hline 3 Luis de Belmonte & 24 & 548897 & 0,200144 \\
\hline 4 Sebastián de Villaviciosa & 23 & 155324 & 0,66766 \\
\hline 5 Agustín Moreto & 21 & 313696 & 0,655214 \\
\hline 6 Francisco de Rojas Zorrilla & 18 & 205765 & 0,180931 \\
\hline 7 Antonio Coello & 15 & 166836 & 0,134731 \\
\hline 8 Pedro de Lanini & 13 & 409703 & 0,102587 \\
\hline 9 Luis Vélez de Guevara & 13 & 79101 & 0,139017 \\
\hline 10 Calderón de la Barca & 13 & 55918 & 0,16882 \\
\hline 11 Juan de Zabaleta & 12 & 14360 & 0,290428 \\
\hline 12 Antonio Martínez de Meneses & 12 & 31047 & 0,279733 \\
\hline 13 Juan de Vélez de Guevara & 8 & 64722 & 0,169175 \\
\hline 14 Diego de Figueroa & 7 & 0 & 0,10812 \\
\hline 15 José de Figueroa & 7 & 0 & 0,10812 \\
\hline 16 Antonio Mira de Amescua & 7 & 29269 & 0,48536 \\
\hline 17 Pedro Rosete & 7 & 1485 & 0,17582 \\
\hline 18 Juan Bautista Diamante & 7 & 0 & 0,228654 \\
\hline 19 Francisco de Avellaneda & 7 & 0 & 0,246749 \\
\hline 20 Francisco Villegas & 5 & 103 & 0,051259 \\
\hline 21 José de Cañizares & 4 & 52 & 0,007854 \\
\hline 22 Juan de la Hoz y Mota & 4 & 52 & 0,007854 \\
\hline 23 Antonio de Huerta & 3 & 0 & 0,62511 \\
\hline 24 Juan Pérez de Montalbán & 3 & 52 & 0,131124 \\
\hline 25 Antonio Solís & 3 & 103 & 0,008439 \\
\hline 26 Antonio de Vargas & 2 & 52 & 0,010892 \\
\hline 27 Nicolás Villarroel & 2 & 0 & 0,010872 \\
\hline
\end{tabular}




\begin{tabular}{|c|c|c|c|}
\hline LABEL & Degree Centrality & Betweenness Centrality & Eigenvector Centrality \\
\hline 28 Diego de Villegas & 2 & 52 & 0,010892 \\
\hline 29 Guillén de Castro & 2 & 0 & 0,013467 \\
\hline 30 Antonio Zamora & 2 & 0 & 0,002148 \\
\hline 31 Manuel León Marchante & 2 & 0 & 0,001258 \\
\hline 32 Diego Calleja & 2 & 0 & 0,001258 \\
\hline 33 Juan Coello Arias & 2 & 0 & 0,36303 \\
\hline 34 Jusepe Rojo & 2 & 0 & 0,005752 \\
\hline 35 Antonio de Bances Candamo & 2 & 0 & 0,002148 \\
\hline 36 Juan Maldonado & 2 & 1 & 0,001063 \\
\hline 37 Juan de Vera Tassis & 2 & 1 & 0,001063 \\
\hline 38 Andrés Gil Enríquez & 2 & 0 & 0,087113 \\
\hline 39 Francisco González & 1 & 0 & 0,005436 \\
\hline 40 Juan Luis Alarcón & 1 & 0 & 0,010342 \\
\hline 41 Fernando de Ludena & 1 & 0 & 0,010342 \\
\hline 42 Jacinto de Herrera & 1 & 0 & 0,010342 \\
\hline 43 Francisco de Tapia & 1 & 0 & 0,010342 \\
\hline 44 Pedro de Leyva & 1 & 0 & 0,000524 \\
\hline 45 Pedro Correa & 1 & 0 & 0,000524 \\
\hline 46 Sor Juana Inés de la Cruz & 1 & 0 & 0,007907 \\
\hline 47 José de Bolea & 1 & 0 & 0,000524 \\
\hline 48 Francisco de la Torre & 1 & 0 & 0,000524 \\
\hline 49 Isidro de Burgos Mantilla & 1 & 0 & 0,000536 \\
\hline 50 Alfonso Alfaro & 1 & 0 & 0,010342 \\
\hline 51 Antonio Sigler de Huerta & 1 & 0 & 0,010342 \\
\hline 52 Diego La Dueña & 1 & 0 & 0,000613 \\
\hline 53 Jerónimo Cifuentes & 1 & 0 & 0,000613 \\
\hline 54 Antonio Hurtado de Mendoza & 1 & 0 & 0,000613 \\
\hline 55 Diego Vera Ordóñez & 1 & 0 & 0,000524 \\
\hline 56 José de Ribera & 1 & 0 & 0,000524 \\
\hline 57 Román Montero de Espinosa & 1 & 0 & 0,002876 \\
\hline 58 Luis de Ulloa Pereira & 1 & 0 & 0,000524 \\
\hline 59 Rodrigo Dávila & 1 & 0 & 0,000524 \\
\hline 60 José de Luna & 1 & 0 & 0,001073 \\
\hline 61 Vicente Suárez de Deza & 1 & 0 & 0,001073 \\
\hline 62 Francisco Antonio de Monteser & 1 & 0 & 0,001023 \\
\hline 63 Diego de Silva & 1 & 0 & 0,001023 \\
\hline 64 José Ruiz & 1 & 0 & 0,005436 \\
\hline 65 Jacinto Hurtado de Mendoza & 1 & 0 & 0,005436 \\
\hline 66 Agustín Salazar Torres & 1 & 0 & 0,000613 \\
\hline
\end{tabular}




\begin{tabular}{l|c|c|c}
\multicolumn{1}{c|}{ Label } & Degree Centralti & Betweenness Centrality & Eigenvector Centraltiy \\
\hline 67 Lope de Vega Carpio & 1 & 0 & 0,001241 \\
\hline 68 Pedro Álvarez & 1 & 0 & 0,000524 \\
\hline 69 Luis Hurtado de Toledo & 1 & 0 & 0,000524 \\
\hline 70 Lorenzo Daniel & 1 & 0 & 0,000524 \\
\hline 71 Alonso Quadrado & 1 & 0 & 0,000524 \\
\hline 72 Ambrosio Arce & 1 & 0 & 0,043556 \\
\hline 73 Gabriel de Roa & 1 & 0 & 0,009439 \\
\hline 74 Jerónimo de Villanueva & 1 & 0 & 0,009439 \\
\hline 75 Marco Antonio Orí & 1 & 0 & 0,000524 \\
\hline 76 Jacinto Alonso Maluenda & 1 & 0 & 0,000524 \\
\hline
\end{tabular}

Tabla 1. Posición de los dramaturgos colaboradores en relación con la degree centrality, la betweenness centrality y la eigenvector centrality. Fuente: Gephi 0.9.2

Como resultado de la tarea anteriormente descrita es posible ofrecer el siguiente grafo que representa las relaciones de los dramaturgos áureos que participaron en el fenómeno de la composición de las comedias en colaboración. El tamaño de cada nodo representa el grado de importancia de cada uno de los puntos que forma parte de la red y el grueso de las aristas indica el número de colaboraciones que se establece entre dos dramaturgos.

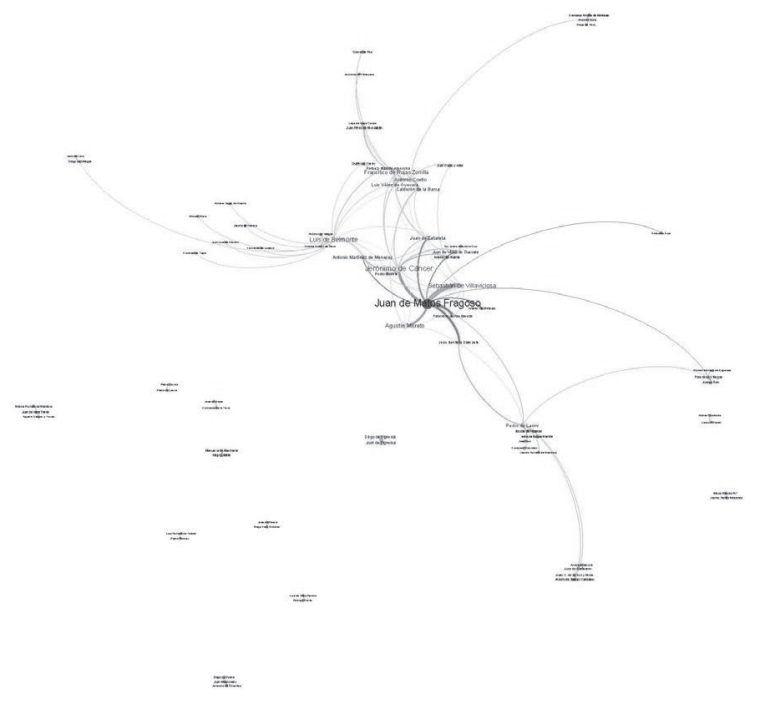

Imagen 1. Grafo que presenta la red completa de dramaturgos colaboradores 


\section{ANÁLISIS Y CONCLUSIONES DE LA RED SOCIAL DE DRAMATURGOS ÁUREOS COLABORADORES}

Este grafo nos ha permitido trazar un diagnóstico general y estático de las relaciones sociales en este contexto. La primera conclusión que es posible apuntar, a partir del grafo, es que nos encontramos ante una red social dispersa en la que observamos diferentes subgrupos. Algunos de ellos, más amplios, centrales y estables, están vinculados entre sí, forman parte de la red y sus miembros tienen relaciones bien cohesionadas. Otros, sin embargo, de carácter bimembre o trimembre, son periféricos, circunstanciales y están desvinculados de la comunidad; así pues, se observa una ausencia de conexiones entre estos y los grupos anteriormente mencionados. Esto nos señala aquellos grupos de dramaturgos que colaboraron de forma más estable y recurrente frente a aquellos que solo lo hicieron de forma puntual.

A este respecto es posible observar en el grafo un total de nueve parejas y dos tríos de dramaturgos que colaboran entre sí pero que están desvinculados de la red. El análisis del grafo los expulsa del sistema por considerar su escasa aportación, lo que demuestra que, en su mayoría, eran aficionados en la elaboración de este tipo de comedias y no formaron parte de la red social mayoritaria. En este aspecto el grafo es revelador de lo que suponía una moda que se llevaba a cabo entre algunos amigos o hermanos pero que, al ser considerados como aficionados, no se incorporaron al negocio teatral de las comedias colaboradas. Se trata de Diego y José de Figueroa; José de Ribera y Diego Vera Ordóñez; Luis Hurtado de Toledo y Pedro Álvarez; Manuel León Marchante y Diego Calleja; José de Bolea y Francisco de la Torre; Pedro Correa y Pedro de Leyva; Antonio Hurtado de Mendoza, Juan de Vera Tassis y Agustín de Salazar y Torres; Marco Antonio Orí y Jacinto Alonso Maluenda; Alonso Cuadrado y Lorenzo Daniel; y, finalmente Diego de La Dueña, Juan Maldonado y Jerónimo de Cifuentes.

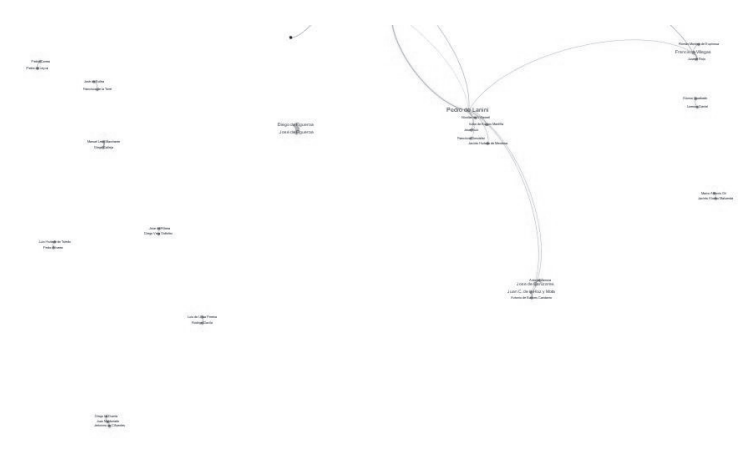




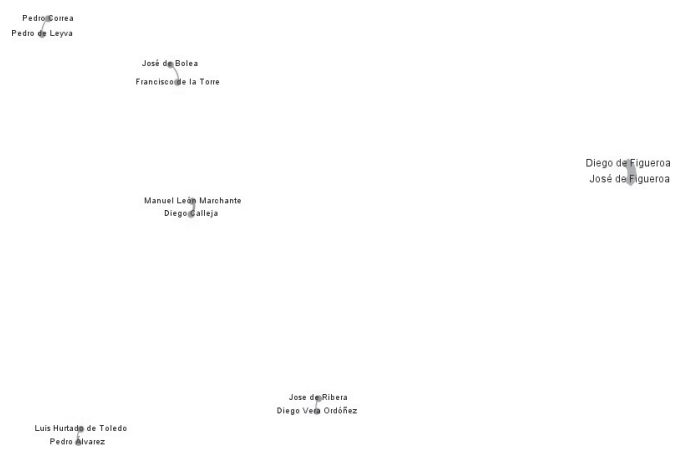

Imagen 2. Segmento del grafo que presenta los grupos de dramaturgos colaboradores periféricos y desvinculados de la red completa

Si nos concentramos en aquel fragmento del grafo cuyos integrantes están vinculados de algún modo, observamos cómo Juan de Matos Fragoso destaca como principal dramaturgo generador de este tipo de comedias. Se trata del dramaturgo que posee el mayor peso y, por tanto, fue el autor que -de acuerdo con los datos que tenemos hasta el momento- escribió mayor número de obras en colaboración. Así pues, constituye el nodo de mayor centralidad de grado (degree centrality), valor que se mide a través del número total de vínculos que tiene cada nodo, es decir, el número total de conexiones o enlaces que tiene cada dramaturgo con los restantes que integran la red. A su vez, el dramaturgo de origen portugués es generador de un grupo social en torno a la elaboración de comedias mancomunadas que, en el conjunto de la red, crea el grupo más cohesionado, lo que lo convierte en el dramaturgo que alcanza una mayor centralidad del vector propio, esto es, se trata de un nodo conectado a muchos nodos que, a su vez, están bien conectados. Es, por tanto, el protagonista del subgrupo principal de los dramaturgos entre los que se encuentran: Jerónimo de Cáncer, Sebastián de Villaviciosa, Agustín Moreto, Antonio Martínez de Meneses, Juan Bautista Diamante, Juan de Zabaleta y Francisco de Avellaneda. Asimismo, parece importante señalar que se trata de una red que no se caracteriza por tener entre sus miembros a los dramaturgos más destacados literariamente, si exceptuamos a Agustín Moreto. 


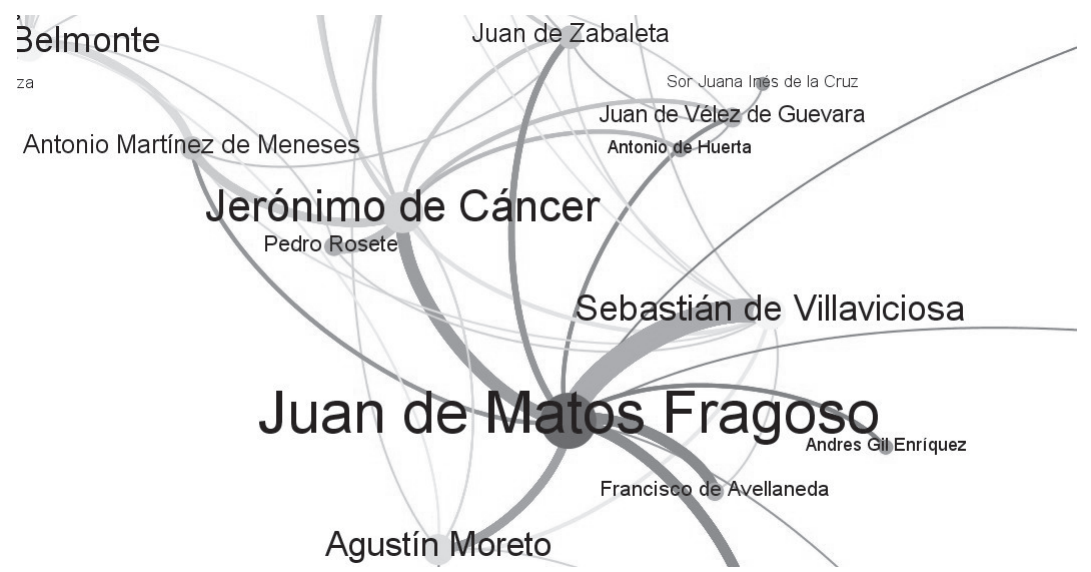

Imagen 3. Segmento del grafo que presenta el nodo de mayor centralidad de la red y el grupo que genera

Sin embargo, aunque Matos sea el dramaturgo con mayor número de obras colaboradas no es el mejor conectado en la red social. Luis Belmonte Bermúdez, otro de los escritores considerado como menores, es el mejor relacionado con todos los subgrupos. Así pues, funciona como el mejor conector (betweenness centrality) y está vinculado con distintas comunidades, pues hace de intermediario entre varios puntos en cuyo camino está situado. Mientras que Matos genera un subgrupo compacto, Belmonte se relaciona con todos los ya existentes, entre los que destacan los generados en torno a Jerónimo de Cáncer y a Francisco de Rojas Zorrilla.

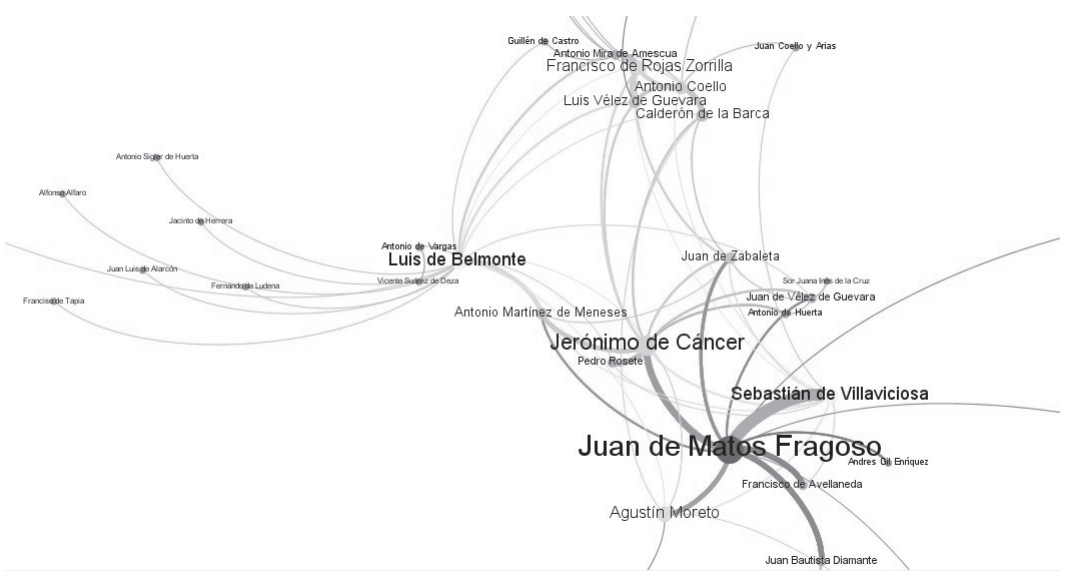




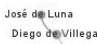

Diego devillegas

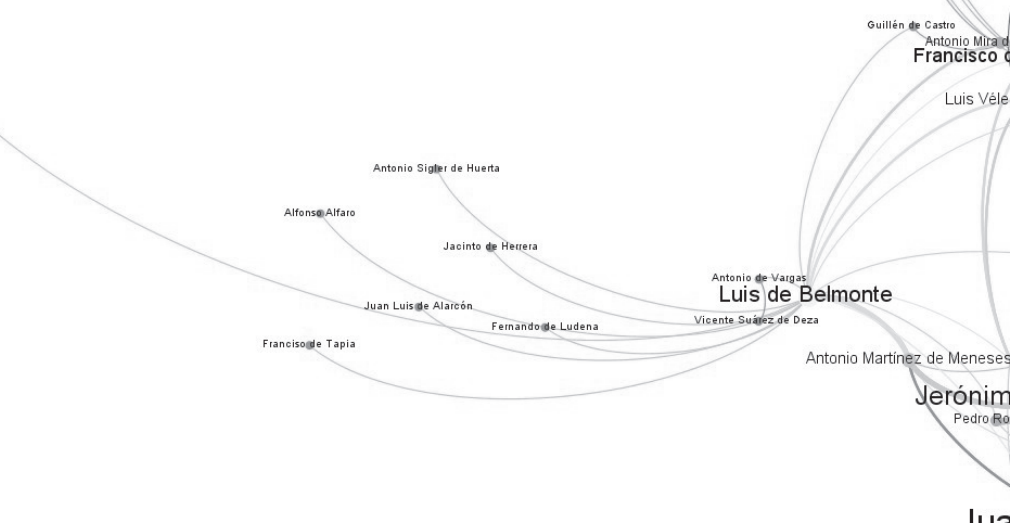

Imagen 4. Segmento del grafo que presenta el nodo que mejor conecta con otros miembros de la red y el grupo que genera

El segundo dramaturgo con mayor peso en cuanto a número de obras en las que colabora es Jerónimo de Cáncer quien, además de generar un subgrupo con Pedro Rosete y Antonio Martínez de Meneses, está plenamente relacionado con el subgrupo de Rojas Zorrilla.

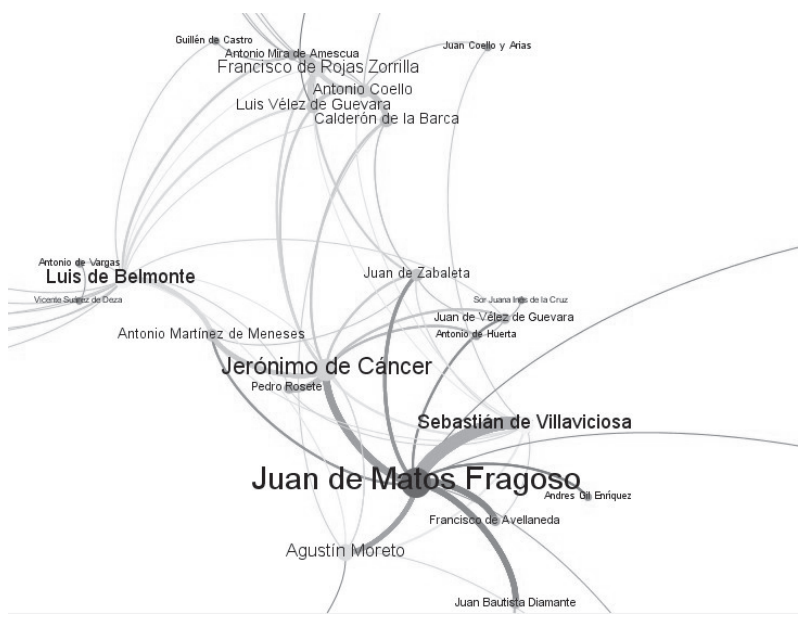

Imagen 5. Segmento del grafo que presenta el nodo que ocupa el segundo puesto en términos de centralidad en la red y el grupo que genera 
El tercer gran subgrupo de interés se genera en torno a Francisco Zorrilla. En él se encuentran una serie de colaboradores más estables o con los cuales se generó un número mayor de colaboraciones como Calderón de la Barca, Antonio Coello, Luis Vélez de Guevara y Mira de Amescua entre otros colaboradores circunstanciales como, por ejemplo, Pérez de Montalbán.

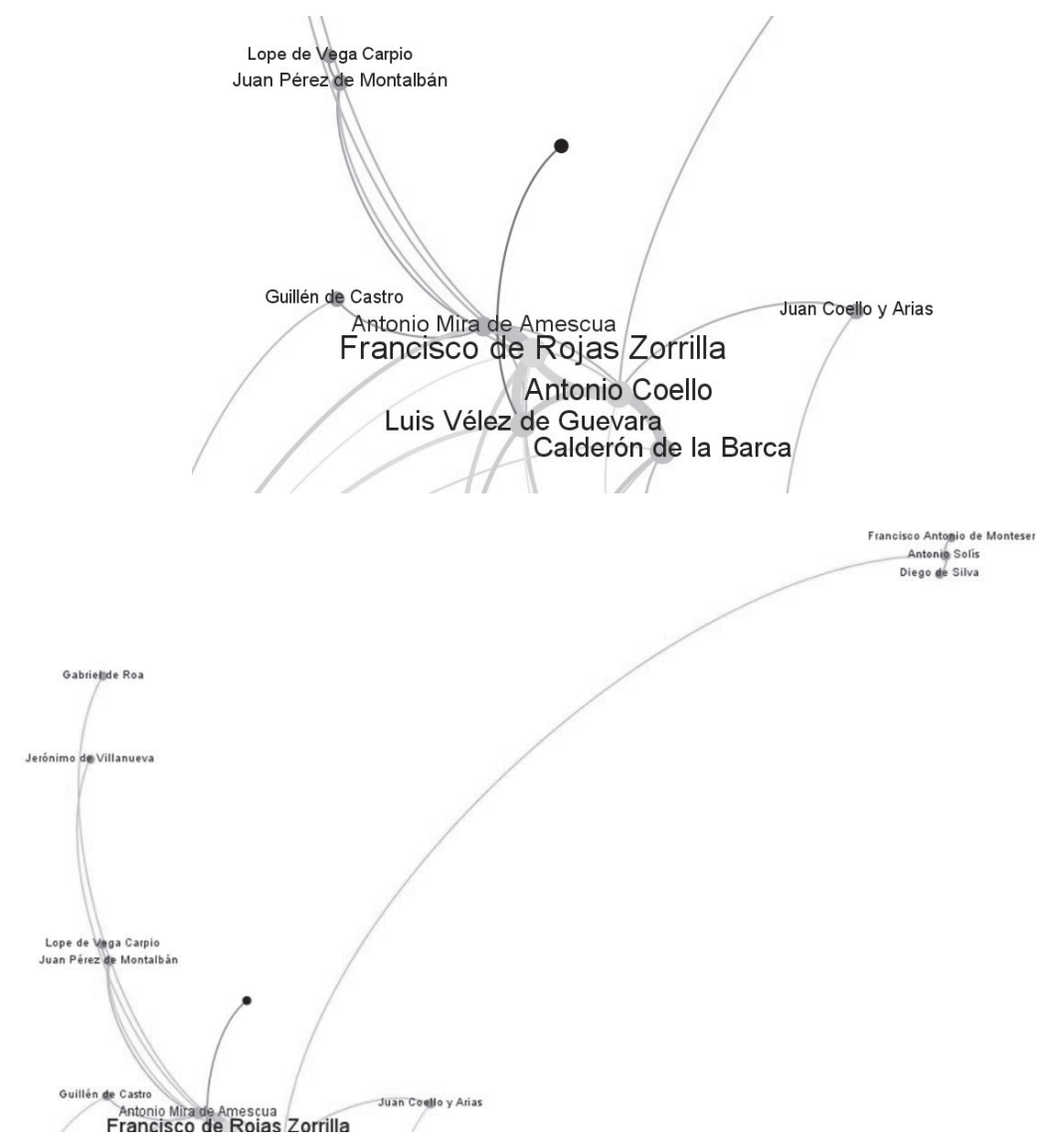

Imagen 6. Segmento del grafo que presenta el nodo que ocupa el tercer puesto en términos de centralidad en la red y el grupo que genera

Un cuarto grupo, de relativa importancia en cuanto al número de colaboraciones, se da en torno a Pedro Lanini. Curiosamente, aunque genera un subgrupo de pequeña entidad, es el tercer dramaturgo mejor relacionado con el resto después de Belmonte y Matos. 

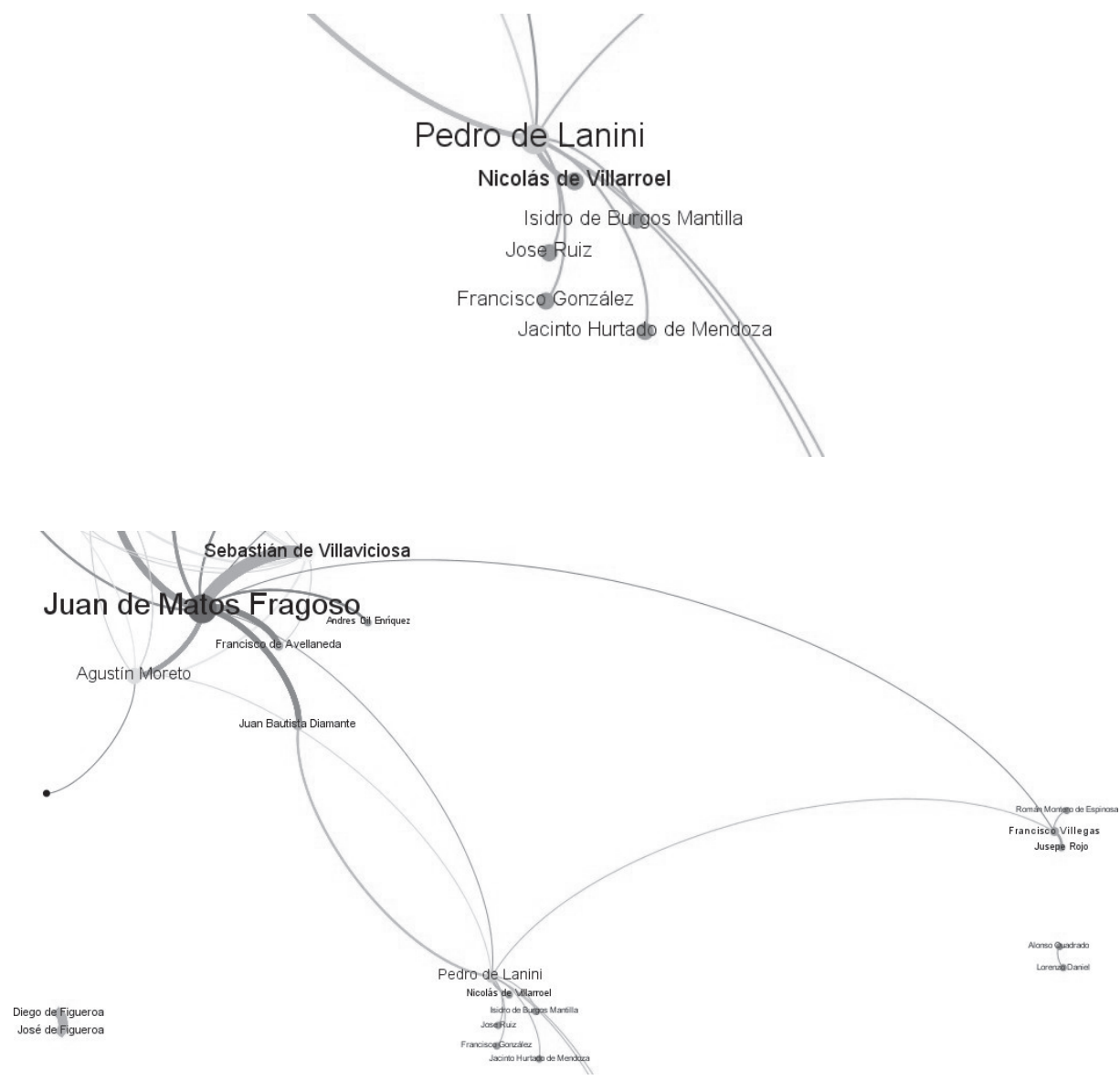

Imágenes 7 y 8 . Segmento de los grafos que presentan el nodo que ocupa el cuarto puesto en términos de centralidad en la red y el grupo que genera

Los restantes grupos que refleja el grafo se sitúan en la periferia de la red, lo que indica una participación menor en el proceso creativo y, por tanto, una relevancia menos significativa en el campo. Como ejemplo es posible mencionar dos grupos bimembres relacionados entre sí que se forman alrededor de Antonio de Bances Candamo y de José de Cañizares. Su posición alejada del centro resulta evidente si consideramos que se trata de dramaturgos cuya producción se sitúa a caballo entre el XVII y el XVIII y que, por tanto, pertenecen a una generación posterior a la calderoniana, en la que es posible emplazar a los dramaturgos principales de la red. 


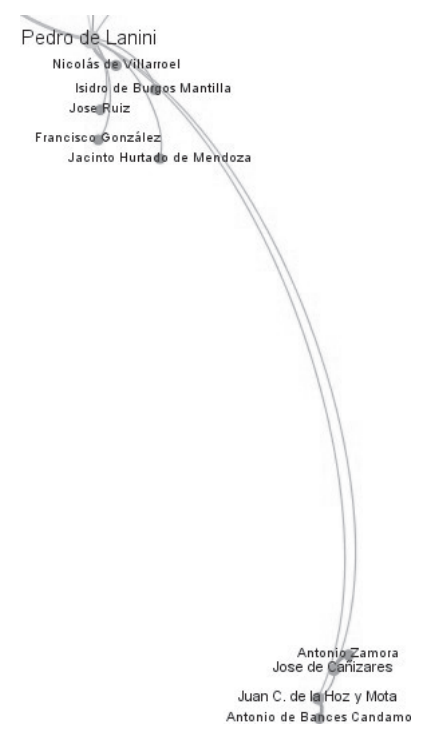

Imagen 9. Segmento del grafo que presenta dos de los grupos bimembres y periféricos de la red

Otro de los grupos periféricos es el que se constituye alrededor de Francisco Villegas y que integran Jusepe Rojo y Román Montero de Espinosa. Esta vez el lugar periférico viene conferido, probablemente, por su participación circunstancial en este modo de composición.

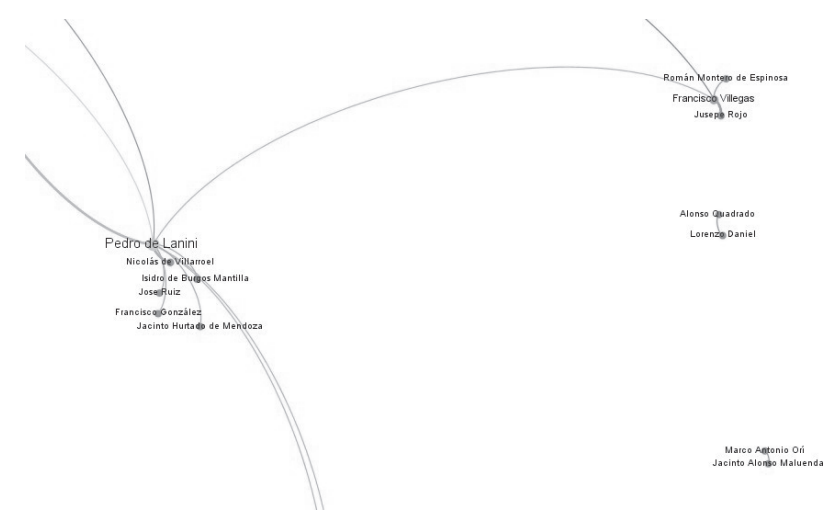

Imagen 10. Segmento del grafo que presenta otro de los grupos trimembres y periféricos de la red 
Uno de los datos más reveladores de este sistema es conocer la lista de los más influyentes en la red que, por orden, son: Luis de Belmonte, Juan de Matos Fragoso, Pedro de Lanini, Agustín Moreto, Francisco de Rojas Zorrilla y Jerónimo de Cáncer. A la vista de este conjunto de dramaturgos parece importante señalar, en primer lugar, la posición de los dramaturgos canónicos en la red. En este sentido, Calderón, más allá de tener un grado muy inferior, por no dedicarse a este tipo de comedias en las que posiblemente colaboró por compromiso, es uno de los dramaturgos con menor influencia en la red, situándose en la posición 13. Algo semejante ocurre con el caso de Lope, que se sitúa en la posición 1; aunque en este caso su baja influencia se debe a que no solo prácticamente no participó en este modo de composición sino que, además, pertenece a una generación anterior y a ello se suma su muerte en 1635. A este respecto puede considerarse que uno de los objetivos futuros en torno a esta investigación consiste en crear grafos diacrónicos que permitan observar la evolución de la red cronológicamente considerando los cambios que en cada década sufrió el campo dramático áureo ibérico.

De forma paralela y, en segundo lugar, parece importante poner de relieve el significado de varios autores, considerados menores por la crítica, en la red social que genera la composición de comedias en colaboración. En este sentido podemos recordar que las últimas décadas has sido prolijas en el acercamiento a los datos biográficos (Martínez Carro; Rubio San Román y Martínez Carro; Martínez Carro y Rubio San Román 2007, 2009, 2015), bibliográficos (Profeti; Profeti y Zancanari) y literarios (Arellano; Cassol y Oteiza) de estos dramaturgos, de los que apenas se conocían noticias por estar eclipsados por las obras y la vida de los grandes maestros. El desinterés que la crítica mostró en los siglos posteriores a sus vidas hacia su producción hizo que la documentación necesaria para su estudio se perdiera y que su obra no siempre se haya considerado en las distintas historias de la literatura o en los proyectos de edición en marcha, al contrario de lo que sucedió con los dramaturgos de primera fila (Arellano 7).

Con respecto al punto editorial es posible recordar, no obstante, cómo en los últimos diez años se han iniciado diversos proyectos que abordan la edición de la obra de algunos de los llamados autores secundarios, lo que, sin duda, permitirá conocer mejor sus textos y, por tanto, situar debidamente a estos autores dentro del campo al que pertenecen tal y como ya Cassol ha señalado (33).

En cualquier caso, los resultados del grafo que ofrecemos y la posición privilegiada de estos dramaturgos en el conjunto de la red social contribuyen 
ahora a la labor de recuperación de sus importantes relaciones amistosas y teatrales, lo que puede funcionar como aliciente para que otros trabajos posteriores aborden su obra desde el punto de vista editorial o bibliográfico. Con esta propuesta hemos pretendido ofrecer un panorama global del conjunto de la red que generaron los dramaturgos áureos participantes en la composición de comedias en colaboración así como de los distintos grupos que forman parte de la red. Este diagnóstico inicial nos ha permitido distinguir la existencia de participantes circunstanciales que no forman parte de la red, frente a un grupo de dramaturgos bien cohesionados de colaboración activa y práctica habitual, entre los cuales destacan de forma significativa algunos de los considerados figuras secundarias o dramaturgos menores, principalmente Matos Fragoso y Belmonte.

Frente a lo planteado cabe preguntarse, en todo caso, hasta qué punto la red expuesta recrea la red del siglo XVII, pues responde al estado actual de las complejas autorías de estas comedias. En este sentido debe recordarse que, a pesar de los avances y esfuerzos antes señalados, las obras de varios de los autores que más destacan en la red apenas cuentan en la actualidad con ediciones críticas, lo que dificulta los trabajos de estilometría. Solo mediante esta disciplina será posible disipar, por una parte, las verdaderas colaboraciones de los autores más olvidados, como, por ejemplo, Matos Fragoso; pero, por otra, probablemente, ayudará también a atribuir nuevas piezas a los dramaturgos canónicos, por ejemplo a Calderón, lo que contribuirá a redefinir sus posiciones en el grafo y, por tanto, a corroborar o a refutar esta propuesta de red social de los dramaturgos colaboradores del Siglo de Oro.

\section{OBRAS CITADAS}

Álvarez Brito, Yaiza, ed. Francisco de Rojas Zorrilla, "El más impropio verdugo". Francisco de Rojas Zorrilla. Obras completas. Segunda parte de comedias. Ed. Instituto Almagro de teatro clásico. Dirs. Felipe Blas Pedraza Jiménez y Rafael González Cañal. Coord. Milagros Rodríguez Cáceres. Vol. 6. Cuenca: Ediciones de la Universidad de Castilla-La Mancha, 2017. 497-750.

Alviti, Roberta. I manoscritti autografi delle commedie del Siglo de Oro scritte in collaborazione. Catalogo e studio. Firenze: Alinea Editrice, 2006.

Arellano, Ignacio, coord. Paraninfos, segundones y epígonos de la comedia del Siglo de Oro. Barcelona: Anthropos, 2004. 
Azam, Martine, y Ainhoa de Federico. "Sociología del arte y análisis de redes sociales". Redes: Revista bispana para el análisis de redes sociales 25.2 (2014): sin pp.

Becerra Mayorga, Witton, y Joice Camacho Machado. "Humanidades Digitales: la censura y los laudatorios en las preliminares del Siglo de Oro español; Madrid y Guzmán de Alfarache”. Hallazgos 13.25 (2016): 111-29.

Bieses (Bibliografía de escritoras españolas). https://www.bieses.net/. Fecha de la consulta: 15/01/2019.

Brown, David M., Adriana Soto-Corominas y Juan Luis Suárez. "The preliminaries Project: geography, networks, and publication in the Spanish Golden Age". Digital Scholarship in the Humanities 32.4.1 (2017): 709-32.

Cassol, Alessandro. "Hijos de un Parnaso menor. Segundones del teatro áureo en las Escogidas (Partes 1-12)". Los segundones. Importancia y valor de su presencia en el teatro aurisecular. Eds. Alessandro Cassol y Blanca Oteiza. Madrid/Frankfurt: Iberoamericana/Vervuert, 2007. 15-34.

Cassol, Alessandro, y Blanca Oteiza, eds. Los segundones. Importancia y valor de su presencia en el teatro aurisecular. Madrid/Frankfurt: Iberoamericana/ Vervuert, 2007.

García Reidy, Alejandro, ed. Agustín Moreto. "La fuerza del natural”. Comedias de Agustín Moreto. Segunda Parte de comedias. Volumen 5. Dir. María Luisa Lobato. Coord. Miguel Zugasti. Kassel: Reichenberger, 2015. 465657.

Gavela, Delia, ed. Agustín Moreto, "El hijo pródigo", Comedias de Agustín Moreto. Alicante: Biblioteca Virtual Miguel de Cervantes, 2019 (Colección Digital PROTEO 11).

González Cañal, Rafael. "La colaboración de Rojas con los hermanos Coello: El robo de las Sabinas". La comedia escrita en colaboración en el teatro del Siglo de Oro. Ed. Juan Matas Caballero. Valladolid: Ediciones Universidad de Valladolid \& Olmedo Clásico, 2017. 113-23.

Hinks, John, y Catherine Feely, eds. Historical Networks in the Book Trade. London and New York: Routledge, 2016.

Isasi, Jennifer. "Acercamiento al análisis del sistema de los personajes en la narrativa escrita en español: el caso de Zumalacárregui y Mendizábal de Pérez Galdós". Caracteres. Estudios culturales y críticos de la esfera digital 6.2 (2017): 107-37.

Mackenzie, Ann L. La escuela de Calderón: estudio e investigación. Liverpool: University Press, 1993. 
Madroñal Durán, Abraham. "La muerte de Valdovinos, de Jerónimo de Cáncer, comedia en colaboración". La comedia escrita en colaboración en el teatro del Siglo de Oro. Ed. Juan Matas Caballero. Valladolid: Ediciones Universidad de Valladolid, 2017. 45-57.

Martínez Carro, Elena. Antonio Martínez de Meneses, vida y obra. Madrid: Fundación Universitaria Española, 2006.

Martínez Carro, Elena, y Alejandro Rubio San Román. "Documentos sobre Jerónimo de Cáncer y Velasco". Lectura y signo: revista de literatura 2.1 (2007): 15-32.

Martínez Carro, Elena, y Alejandro Rubio San Román. "Documentos sobre Jerónimo de Cáncer y su familia (parte II)”. Lectura y signo: revista de literatura 4.1 (2009): 61-77.

Martínez Carro, Elena, y Alejandro Rubio San Román. "Una nota biográfica sobre Jerónimo de Cáncer y Velasco". Revista de Literatura 77.154 (2015): 585-95.

Matas Caballero, Juan, ed. La comedia escrita en colaboración en el teatro del Siglo de Oro. Valladolid: Ediciones Universidad de Valladolid \& Olmedo Clásico, 2017.

Moretti, Franco. Distant Reading. London: Verso, 2013.

Pérez de Montalbán, Juan. Fama póstuma a la vida y muerte del doctor frey Lope Félix de Vega Carpio y elogios panegíricos a la inmortalidad de su nombre. Ed. Enrico Di Pastena. Pisa: Edizioni ETs, 2001.

Profeti, Maria Grazia. Per una bibliografia di fuan Pérez de Montalbán. Verona: Università degli Studi di Padova, 1976.

Profeti, Maria Grazia. Per una bibliografia di Felipe Godinez. Verona: Università degli Studi di Padova, 1982.

Profeti, Maria Grazia, y Umile Maria Zancanari. Per una bibliografia di Alvaro Cubillo de Aragón. Verona: Università degli Studi di Verona, 1983.

Rodríguez-Gallego López, Fernando. "Problemas de autoría en comedias en colaboración: La adúltera penitente, de Matos Fragoso, Cáncer y Moreto”. Boletín de la Real Academia (en prensa).

Rodríguez Treviño, Julio César. "Cómo utilizar el Análisis de Redes Sociales para temas de historia”. Signos Históricos 29 (2013): 102-41.

Rubio San Román, Alejandro, y Elena Martínez Carro. "Relaciones entre Rojas Zorrilla y Jerónimo de Cáncer”. Arbor: Ciencia, pensamiento y cultura 726 (2007): 461-73.

Suárez, Juan Luis. "El humanista digital”. Revista de Occidente 380 (2013): 5-21. 
Trambaioli, Marcella. "La fingida Arcadia de 1666: autoría y escritura de consuno". Moretiana: adversa y próspera fortuna de Agustín Moreto. Coords. María Luisa Lobato López y Juan Antonio Martínez Berbel. Frankfurt: Vervuert / Madrid: Iberoamericana, 2008. 185-208.

Ulla Lorenzo, Alejandra. "Las comedias escritas en colaboración y su publicación en las Partes". Criticón 79-98 (2010): 79-98.

Van Vugt, Ingeborg. "Using Multi-Layared Networks to Disclose Books in the Republic of Letters". Fournal of Historical Network Research 1 (2017): 25-51.

Vega García-Luengos, Germán. "Usos y usufructos de Calderón en las comedias colaboradas". La comedia escrita en colaboración en el teatro del Siglo de Oro. Ed. Juan Matas Caballero. Valladolid: Ediciones Universidad de Valladolid, 2017. 181-202. 